\title{
Spatial Community Structure of Mountain Pine Beetle Fungal Symbionts Across a Latitudinal Gradient
}

\author{
Amanda D. Roe • Patrick M. A. James • \\ Adrianne V. Rice • Janice E. K. Cooke • \\ Felix A. H. Sperling
}

Received: 30 November 2010 / Accepted: 28 February 2011 /Published online: 6 April 2011

(C) The Author(s) 2011. This article is published with open access at Springerlink.com

\begin{abstract}
Symbiont redundancy in obligate insect-fungal systems is thought to buffer the insect host against symbiont loss and to extend the environmental conditions under which the insect can persist. The mountain pine beetle is associated with at least three well-known and putatively obligate ophiostomatoid fungal symbionts that vary in their environmental tolerances. To better understand the spatial variation in beetle-fungal symbiotic associations, we examined the community composition of ophiostomatoid fungi associated with the mountain pine beetle as a function of latitude and elevation. The region investigated represents the leading edge of a recent outbreak of mountain pine beetle in western Canada. Using regression and principal components analysis, we identified significant spatial patterns in fungal species abundances that indicate symmetrical replacement between two of the three fungi along a latitudinal gradient and little variation in response to elevation. We also identified significant variation in the prevalence of pair-wise species combinations that occur within beetle galleries. Frequencies of pair-wise combinations were significantly different from what was expected given overall species abundances. These results suggest that complex processes of competitive exclusion and coexistence help determine fungal community composition and
\end{abstract}

Amanda D. Roe and Patrick M. A. James have joint first authorship.

Electronic supplementary material The online version of this article (doi:10.1007/s00248-011-9841-8) contains supplementary material, which is available to authorized users.

A. D. Roe $(\bowtie) \cdot$ P. M. A. James $\cdot$ A. V. Rice · J. E. K. Cooke F. A. H. Sperling

Department of Biological Sciences, University of Alberta,

Edmonton, AB, Canada

e-mail: amandaroe5@gmail.com that the consequences of these processes vary spatially. The presence of three fungal symbionts in different proportions and combinations across a wide range of environmental conditions may help explain the success of mountain pine beetle attacks across a broad geographic range.

\section{Introduction}

The study of symbioses is fertile ground for the exploration of ecological and evolutionary relationships between intimately associated organisms $[1,2]$. Bark beetles in the genus Dendroctonus and their mutualistic ectosymbionts are well-known models of symbioses [1,3]. One member of this group, the mountain pine beetle (MPB; Curculionidae: Scolytinae: Dendroctonus ponderosae (Hopkins)), is of particular interest for its complex multipartite symbioses and capacity for large-scale population irruptions [4]. Recent outbreaks and population expansions of MPB have resulted in broad-scale and unexpected damage to forests in western North America [5, 6]. The unprecedented scale, extent, and intensity of this recent outbreak highlight the need to better understand the MPB system and the interactions between the MPB and its fungal symbionts.

MPBs are known for their close association with a number of symbiotic fungal species that often coexist in multipartite relationships [7]. Fungi in the family Ophiostomataceae are the most well known and play a number of beneficial roles for the beetle host by providing nutrition, protection against tree defenses, and modifying environmental conditions within the tree [8-11]. While MPBs are known to carry a diverse microsymbiont fauna, the main ophiostomatoid associates in western Canada are Grosmannia clavigera (Robinson-Jeffrey and Davidson) Zipfel, de Beer, and Wingfield; Leptographium longiclavatum Lee, Kim, and 
Breuil; and Ophiostoma montium (Rumbold) von Arx [12, 13].

Given the importance of fungi to MPB survival and reproduction [8,11, 14-16], a number of studies have focused on teasing apart the interactions among fungal symbionts and the MPB host. Previous studies have demonstrated that the abundance and composition of fungal symbionts associated with MPB vary in both space and time [7, 17, 18]. Presence and relative abundance of different symbionts are affected by environmental conditions, in particular temperature [7, 17, 19]. Specifically, $G$. clavigera has a lower optimal growth temperature than $O$. montium, and consequently is more abundant in cooler conditions than the latter $[7,20]$. L. longiclavatum similarly becomes more abundant in northern climates, such as northwestern Alberta, while it decreases in abundance in southern latitudes [18], despite having a growth optimum similar to $G$. clavigera [13, 19]. Studies have also demonstrated that two MPB fungal symbionts, $G$. clavigera and $O$. montium, can coexist despite apparent symbiont redundancy $[7,21]$.

Given that the relative abundance of fungal symbionts can change across a study region and that these shifts are likely to affect MPB fitness [16], we sought to examine patterns of spatial and temporal heterogeneity in MPB fungal symbiont community composition at the northeastern edge of the current outbreak. We examined coexistence and competition among fungal species at regional spatial scales and further explored how this variation translated into landscape-level variation in fungal assemblages [22]. Until now, the majority of research has focused on MPB populations in northwestern USA and southern British Columbia (BC). However, the population expansion associated with the current outbreak is occurring in northern British Columbia and Alberta (AB) where less is known about patterns of fungal symbiont association and community composition.

To better understand the nature of symbiotic beetle-fungus associations at the northern and eastern edges of the MPB outbreak, we sampled 50 sites distributed along a latitudinal gradient in eastern British Columbia and western Alberta over the course of two MPB flights. This latitudinal gradient is strongly associated with a gradient in elevation. Given the combined influence of elevation and latitude on environmental conditions [23], we also examined the role of elevation in determining community composition. Our objective was to describe the biogeographic patterns of fungal community composition in this region. We focused on three specific questions: (1) How does fungal abundance and species composition vary along this latitudinal gradient? (2) Under what conditions do all three species (G. clavigera, $O$. montium, and L. longiclavatum) coexist and under which conditions are some species excluded? and (3)What degree of temporal variation do we observe between years in fungal abundance and species composition? Our broad-scale approach seeks to identify landscape-level processes that affect MPB symbiont composition, and this study represents one of the first to examine these community patterns using large-scale surveys of natural MPB populations. From previous work on fungal temperature tolerances [7, 19], we expected that the abundance of $G$. clavigera and $O$. montium would decrease with increasing latitude, with an opposing trend observed in L. longiclavatum. Furthermore, we expected to see temporal variation between years, given the temperature sensitivity of fungal growth.

\section{Methods}

\section{Data Collection}

A nested sampling design with three levels was used to survey fungal composition in regions with large numbers of newly, successfully attacked pine trees. Trees were identified by provincial ground or aerial surveys in eastern $\mathrm{BC}$ and western $\mathrm{AB}$, respectively (Fig. 1; Table 1; Electronic Supplementary Material 1), as previously described in Roe et al. [24, 25]. Sampling occurred during two time periods, February-May 2007 (period 1) and September 2007-April 2008 (period 2), which represent independent dispersal flights of MPB during the 2006 and 2007 seasons, respectively. The three levels to this sampling design were (1) landscape, (2) sites within landscapes, and (3), individual trees within sites (Fig. 1; Electronic Supplementary Material 1). Landscapes were selected to represent a range of ecoregions [26] throughout the leading edge of the MPB outbreak, with up to five sites sampled in each landscape at a minimum $5 \mathrm{~km}$ apart (Table 1). Within each site up to ten green attacked trees (minimum $50 \mathrm{~m}$ apart) were sampled. Green attacked trees are those that show recent evidence of MPB attack, contain developing brood, and whose needles have not yet turned red or gray. From each tree, eight phloem + xylem disks containing sections of MPB galleries were obtained using a $10-\mathrm{cm}$ hole saw and chisel. Disks were sampled systematically, with four disks removed at $0.5-1.0 \mathrm{~m}$ and the remaining disks removed at $1.3-1.6 \mathrm{~m}$ in the orientation shown in Electronic Supplementary Material 1. Disks were transported on ice back to the laboratory for fungal culturing.

\section{Fungal Culturing}

Fungi were cultured from larval and adult (parental) MPB as well as from wood samples taken adjacent to individual MPB within galleries following the protocol described in Roe et al. [25]. A single gallery was sampled per disk to 


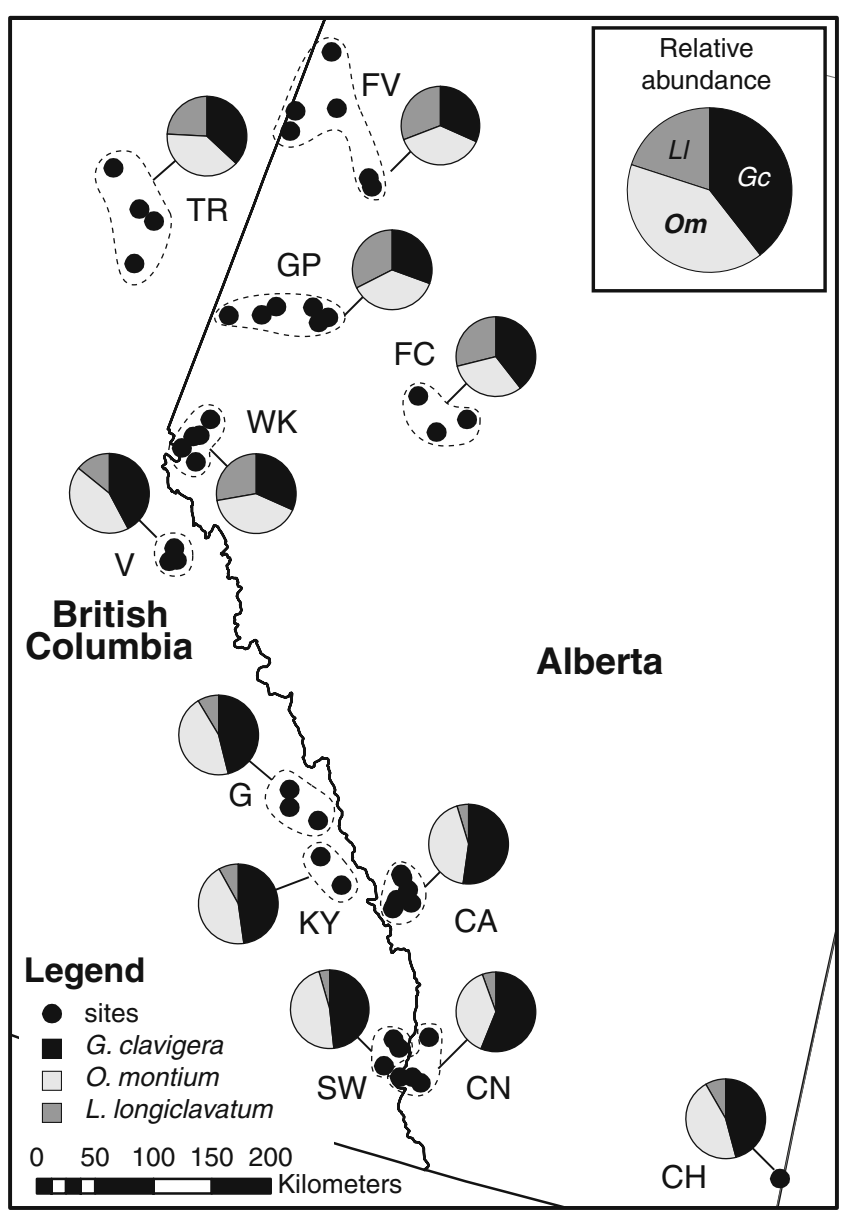

Figure 1 Location of collection sites for MPB fungal symbionts and the relative abundance of each fungal species scored in the 12 landscapes. Inset shows overall relative abundance; Gc, G. clavigera; Om, O. montium; $\mathrm{Ll}, \mathrm{L}$. longiclavatum. Landscapes, with one to six sites, as follows: $C A$, Canmore; $C H$, Cypress Hills; $C N$, Crowsnest Pass; $F C$, Fox Creek; FV, Fairview; G, Golden; GP, Grande Prairie; $K Y$, Kootenay-Yoho; $S W$, Sparwood; $T R$, Tumbler Ridge; $V$, Valemount; $W K$, Willmore-Kakwa

ensure that independent galleries were sampled, resulting in two isolations per parental gallery (i.e., one from the insect and one from an adjacent wood sample). Isolations were stored at room temperature and ambient light, and colonies were subcultured as they appeared (2-5 days, subculturing occurred most often at day 3). Colonies of the three most common fungal associates tended to appear within 2-3 days at room temperature [19], and isolation of individual colonies after longer incubation periods became problematic due to overgrowth by faster-growing species, as well as contamination by mites, bacteria, and other fungi (e.g., yeasts). Sub-cultures were plated onto malt extract agar and then incubated for 2-4 weeks at similar conditions before being scored for the presence of fungal associates. Individual species were identified based on morphological and microscopic traits of known MPB fungal associates, which were derived from original species descriptions and authenticated strains, as described in Roe et al. [25]. A small number of strains were morphologically ambiguous and were termed "intermediate." Detailed descriptions of cultural and microscopic morphological traits used for identification are provided (Electronic Supplementary Material 2).

Given the uniform temperature and incubation period used in this methodology, it is possible that slowergrowing, rare, or cryptic species were missed in these mixed-species cultures, favoring the identification of fastergrowing, dominant species. To assess the success rate of morphotyping, identifications based on multilocus sequence typing (MLST) genotype data (GenBank GU370130GU370344, HQ413347-HQ413650) for a subset of strains $(n=493)$ were contrasted with their initial morphotyping diagnoses. The methodologies and resulting multilocus sequence typing data were originally published in Roe et al. [24, 25], but an abbreviated summary is provided here. Representative strains were deposited in the University of Alberta Microfungus Collection and Herbarium (UAMH 10965-10970, 11000-11007, 11013-11020, 11035-11084, 11105-11119, 11134-11149), as described in Roe et al. [24, 25].

\section{Analyses}

We calculated the relative abundance of each of the three fungal species and species composition within individual galleries. Relative abundance describes the proportion of individuals scored as a species relative to the total number of isolates screened. Species composition describes the combination of species that were simultaneously found within an individual gallery. In this system, there are eight species combinations possible: G. clavigera alone, $O$. montium alone, and L. longiclavatum alone; paired combinations G. clavigera-O. montium, G. clavigera-L. longiclavatum, and O. montium-L. longiclavatum; all three in combination, G. clavigera-O. montium-L. longiclavatum; and zero species. We used the proportions of each of three combinations to describe changes in the fungal community in space and time. Relative abundance and composition were summarized at the level of sites nested within landscapes for a total of 50 sample sites within 12 landscapes (Table 1, Fig. 1). Given the spatial extent of the study area, we did not examine tree level variation and instead focus on broad biogeographic patterns.

\section{Relative Abundance}

We used principal components analysis (PCA) to visualize variation among sites in fungal community composition and to identify which species drive differentiation among sites. Ordination using PCA reduces the dimensionality of multivariate data, exposes intrinsic gradients, and allows 
Table 1 Localities surveyed for mountain pine beetle fungal symbionts

\begin{tabular}{|c|c|c|c|c|c|c|c|c|c|}
\hline Landscape & Province & Site & Period $^{\mathrm{a}}$ & Lat $^{\mathrm{b}}$ & Long $^{\mathrm{b}}$ & Elevation (m) & Eco. ${ }^{\mathrm{c}}$ & Eco. name ${ }^{c}$ & No. of trees \\
\hline \multirow[t]{7}{*}{ Fairview } & \multirow[t]{7}{*}{$\mathrm{AB}$} & FV.a & 1 & 56.128 & -118.548 & 635 & 138 & Peace Lowland & 10 \\
\hline & & FV.b & 1 & 56.314 & -119.892 & 721 & 138 & Peace Lowland & 7 \\
\hline & & FV.c & 1 & 56.182 & -118.633 & 642 & 138 & Peace Lowland & 6 \\
\hline & & FV.aa & 2 & 56.599 & -119.384 & 949 & 137 & Clear Hills Upland & 10 \\
\hline & & FV.bb & 2 & 56.158 & -118.643 & 639 & 138 & Peace Lowland & 10 \\
\hline & & FV.cc & 2 & 56.991 & -119.738 & 792 & 137 & Clear Hills Upland & 10 \\
\hline & & FV.dd & 2 & 56.469 & -119.932 & 748 & 137 & Clear Hills Upland & 10 \\
\hline \multirow[t]{4}{*}{ Tumbler Ridge } & \multirow[t]{4}{*}{$\mathrm{BC}$} & TR.aa & 2 & 55.539 & -121.985 & 842 & 200 & Central Can Rocky Mts & 8 \\
\hline & & TR.bb & 2 & 54.913 & -121.230 & 902 & 200 & Central Can Rocky Mts & 10 \\
\hline & & TR.cc & 2 & 55.321 & -121.441 & 942 & 200 & Central Can Rocky Mts & 10 \\
\hline & & TR.dd & 2 & 55.275 & -121.191 & 1,012 & 200 & Central Can Rocky Mts & 7 \\
\hline \multirow[t]{6}{*}{ Grande Prairie } & \multirow[t]{6}{*}{$\mathrm{AB}$} & GP.a & 1 & 55.088 & -118.756 & 579 & 138 & Peace Lowland & 10 \\
\hline & & GP.b & 1 & 55.056 & -118.510 & 632 & 138 & Peace Lowland & 10 \\
\hline & & GP.c & 1 & 54.995 & -119.222 & 683 & 138 & Peace Lowland & 10 \\
\hline & & GP.aa & 2 & 54.992 & -118.614 & 607 & 138 & Peace Lowland & 7 \\
\hline & & GP.bb & 2 & 54.899 & -119.369 & 764 & 143 & W Boreal & 11 \\
\hline & & GP.cc & 2 & 54.803 & -119.789 & 888 & 143 & W Boreal & 10 \\
\hline \multirow[t]{4}{*}{ Fox Creek } & \multirow[t]{4}{*}{$\mathrm{AB}$} & FC.a & 1 & 54.703 & -117.021 & 708 & 143 & W Boreal & 8 \\
\hline & & FC.b & 1 & 54.651 & -116.301 & 987 & 145 & W AB Upland & 7 \\
\hline & & FC.c & 1 & 54.480 & -116.635 & 862 & 143 & W. Boreal & 6 \\
\hline & & FC.aa & 2 & 54.481 & -116.635 & 786 & 143 & W. Boreal & 10 \\
\hline \multirow[t]{6}{*}{ Willmore-Kakwa } & \multirow[t]{6}{*}{$\mathrm{AB}$} & WK.a & 1 & 53.713 & -119.743 & 1,664 & 207 & E Continental Ranges & 10 \\
\hline & & WK.b & 1 & 53.776 & -119.761 & 1,866 & 207 & E Continental Ranges & 9 \\
\hline & & WK.c & 1 & 53.826 & -119.657 & 1,439 & 207 & E Continental Ranges & 10 \\
\hline & & WK.aa & 2 & 53.996 & -119.526 & 1,622 & 207 & E Continental Ranges & 10 \\
\hline & & WK.bb & 2 & 53.650 & -119.509 & 1,377 & 207 & E Continental Ranges & 10 \\
\hline & & WK.cc & 2 & 53.854 & -119.584 & 1,547 & 207 & E Continental Ranges & 10 \\
\hline \multirow[t]{3}{*}{ Valemount } & \multirow[t]{3}{*}{$\mathrm{BC}$} & V.aa & 2 & 52.882 & -119.301 & 847 & 213 & S Rocky Mtn Trench & 10 \\
\hline & & V.bb & 2 & 52.853 & -119.382 & 1,164 & 213 & S Rocky Mtn Trench & 10 \\
\hline & & V.cc & 2 & 52.963 & -119.380 & 898 & 206 & W Continental Ranges & 10 \\
\hline \multirow[t]{3}{*}{ Golden } & \multirow[t]{3}{*}{$\mathrm{BC}$} & G.aa & 2 & 51.074 & -116.382 & 1,330 & 206 & W Continental Ranges & 11 \\
\hline & & G.bb & 2 & 51.361 & -116.937 & 1,679 & 206 & W Continental Ranges & 10 \\
\hline & & G.cc & 2 & 51.495 & -117.003 & 1,494 & 206 & W Continental Ranges & 10 \\
\hline \multirow[t]{2}{*}{ Kootenay/Yoho } & \multirow[t]{2}{*}{$\mathrm{BC}$} & KY.aa & 2 & 50.912 & -116.037 & 1,234 & 206 & W Continental Ranges & 10 \\
\hline & & KY.bb & 2 & 51.334 & -116.550 & 1,190 & 206 & W Continental Ranges & 10 \\
\hline \multirow[t]{7}{*}{ Canmore } & \multirow[t]{7}{*}{$\mathrm{AB}$} & CA.a & 1 & 50.859 & -115.349 & 1,906 & 207 & E Continental Ranges & 10 \\
\hline & & CA.b & 1 & 51.111 & -115.352 & 1,691 & 214 & N Continental Divide & 10 \\
\hline & & CA.aa & 2 & 50.932 & -115.336 & 2,054 & 207 & E Continental Ranges & 10 \\
\hline & & CA.bb & 2 & 50.940 & -115.153 & 1,579 & 214 & N Continental Divide & 10 \\
\hline & & CA.cc & 2 & 51.030 & -115.243 & 1,426 & 214 & N Continental Divide & 10 \\
\hline & & CA.dd & 2 & 50.877 & -115.347 & 1,953 & 207 & E Continental Ranges & 10 \\
\hline & & CA.ee & 2 & 51.128 & -115.380 & 1,382 & 214 & N Continental Divide & 14 \\
\hline \multirow[t]{3}{*}{ Sparwood } & $\mathrm{BC}$ & SW.aa & 2 & 49.680 & -114.911 & 1,321 & 214 & N Continental Divide & 9 \\
\hline & & SW.bb & 2 & 49.840 & -114.801 & 1,610 & 214 & N Continental Divide & 10 \\
\hline & & SW.cc & 2 & 49.894 & -114.895 & 1,312 & 214 & N Continental Divide & 9 \\
\hline Crowsnest Pass & $\mathrm{AB}$ & CN.a & 1 & 49.629 & -114.696 & 1,422 & 214 & N Continental Divide & 7 \\
\hline & & CN.aa & 2 & 49.657 & -114.552 & 1,510 & 214 & N Continental Divide & 3 \\
\hline & & CN.bb & 2 & 49.986 & -114.495 & 1,697 & 214 & N Continental Divide & 2 \\
\hline & & CN.cc & 2 & 49.629 & -114.434 & 1,548 & 214 & N Continental Divide & 5 \\
\hline
\end{tabular}


Table 1 (continued)

\begin{tabular}{|c|c|c|c|c|c|c|c|c|c|}
\hline Landscape & Province & Site & Period $^{\mathrm{a}}$ & $\mathrm{Lat}^{\mathrm{b}}$ & Long $^{\mathrm{b}}$ & Elevation (m) & Eco. ${ }^{\mathrm{c}}$ & Eco. name ${ }^{c}$ & No. of trees \\
\hline Cypress Hills & $\mathrm{AB}$ & CH.aа & 2 & 49.593 & -110.036 & 1,357 & 160 & Cypress Upland & 6 \\
\hline
\end{tabular}

such data to be interpreted diagrammatically [27]. Prior to analysis, the community data (abundance data pooled by site) were converted to relative abundance of the three species at each site and standardized using the Hellinger transformation [28]. PCA was then performed on the total set of standardized data using the $r d a$ function of the vegan package in R [29]. Significant PCA axes were identified using the broken stick criterion [30]. Following PCA, we fit vectors of latitude and elevation onto the ordination space to identify which axes and which species were most strongly correlated with the different gradients. Post-hoc vector fitting was done using the envfit function in the vegan package in $\mathrm{R}$.

\section{Rate of Change}

Three linear mixed-effects regression models were used to describe the relationship of each species' relative abundance to changes in latitude and elevation. Latitude and elevation were used as fixed effects while sample landscape was used as a random effect to account for additional landscape specific variation. Fixed-effect predictor variables were standardized to zero mean and unit variance prior to analysis. Regression models for all three species were significantly improved by the inclusion of the landscape random effect (data not shown). Three hypotheses based on previous research [e.g., 7, 17, 18] were tested using these models: (1) G. clavigera will decrease in relative abundance with increases in latitude, (2) O. montium will also decrease in relative abundance with increases in latitude, and (3) L. longiclavatum will increase in relative abundance with increases in latitude. Specific hypotheses regarding elevation were not tested because of the high degree of correlation with latitude and the lack of a consistent influence of elevation on fungal abundances as identified using the regression models described above. Analysis of covariance (ANCOVA) was then used to compare the rates of change (i.e., slopes) among pairs of the three fungal species in response to latitude. In particular, we were interested in ascertaining whether the rate of change in the abundance of $G$. clavigera from north to south was significantly different from the rate of change of $L$. longiclavatum from south to north. Because the rate of change in $G$. clavigera is negative, while that of $L$. longiclavatum is positive, we used the absolute value of the slope of L. longiclavatum for comparison (i.e., converted values to have a negative slope). The pair-wise ANCOVA tests used latitude and fungal species as predictor variables, and tested for a significant interaction effect between them. As in the single-species models described above, sample landscape was used as a random effect. All mixed-effect models were carried out using the lme function in the nlme package in $\mathrm{R}[31,32]$ and were summarized using marginal sums of squares.

\section{Differences Between Years}

Because fungi were sampled over two sampling periods, we also compared relative fungal abundance in period 1 and period 2 to identify potential temporal variation. Similar to comparisons among the different species and their response to latitude, we used ANCOVA to test whether sampling period affected relative species abundance.

\section{Species Coexistence}

We used multiple Chi-square tests to test patterns of fungal species coexistence using species composition within galleries as a measure of coexistence. Specifically, we assessed the significance of differences in the proportions of each of the three pair-wise species combinations (i.e., $G$. clavigera-O. montium, G. clavigera-L. longiclavatum, $O$. montium-L. longiclavatum). However, because species varied in relative abundances, the differences in the proportions of each combination could be simply due to the varying probability of species co-occurrence. We also used a series of Chi-square tests to assess whether the observed occurrence of each species combination was different than expected given individual species abundances. In these tests, the null hypothesis was that the frequency of each pair-wise combination was proportional to the product of their individual abundances. Tests were conducted for the entire study area as well as for the 
northern and southern groups of sites. All statistical analyses were conducted in R [33].

\section{Results}

Over the two sampling periods, 2,852 MPB galleries were surveyed in 50 sites from 12 landscapes (Fig. 1, Table 1). Landscapes spanned $7.5^{\circ}$ of latitude, from $49.5^{\circ}$ to $57.0^{\circ} \mathrm{N}$. Based on MPB distribution patterns, these landscapes formed two natural groups that matched the MPB distribution in the region: a northern group (Fairview, Tumbler Ridge, Grande Prairie, Fox Creek, Wilmore-Kakwa, and Valemount) and a southern group (Golden, KootenayYoho, Canmore, Sparwood, Crowsnest Pass, and Cypress Hills). During the sampling periods, no MPBs were detected in the intervening area between Valemount, $\mathrm{BC}$ and Golden, BC. These groups also correspond to broadscale phylogeographic patterns previously detected in $G$. clavigera, O. montium, and L. longiclavatum [24]. The number of sites surveyed per landscape ranged from one to seven. The majority of sites were located in lodgepole pine stands (Pinus contorta Douglas var. latifolia Engelmann), with the exception of Fox Creek, which was in a putative $P$. contorta Douglas var. latifolia Engelmann $\times$ Pinus banksiana Lamb. hybrid zone. The extent of this zone of hybridization is not well defined [34, and the references therein]. Since hybrids were determined morphologically, it is conceivable that trees of undiagnosed hybrid status were also sampled. Sampling between the two time periods was disproportionate. In period 1, 599 galleries were surveyed at 15 sites in six landscapes, while in period 2, 2,253 galleries were surveyed at 26 sites in 12 landscapes. Each gallery produced two samples, one from the beetle and one from gallery wood tissue. Given the timing of sampling (winter and early spring), the majority of insect isolates were obtained from larval $(n=2,375)$, rather than adult MPB $(n=557)$. No global differences in fungal species abundance were detected between larval and adult derived fungal samples (data not shown), so larval and adult data were subsequently pooled.

\section{Morphotyping $\times$ Molecular Genotyping Success Rate}

We contrasted initial morphological diagnoses with multilocus sequence data (i.e., MLST) derived identifications for a subset of cultures $(n=493)$ as described in Roe et al. [24, 25]. We identified differences between the morphotyping and genotyping success rates among the three fungal symbionts examined (Electronic Supplementary Material $3)$. Three main results were obtained from these comparisons. First, the overall success rate of morphological diagnosis was $74.6 \%$ (morphological diagnosis equals
MLST genotype), although this success was not evenly distributed between species. Second, two undiagnosed MPB fungal associates were identified through MLST genotyping (Genlisea aurea and Leptographium terebrantis), although together they represent a small proportion $(\sim 5 \%)$ of the subsampled strains. Third, the majority of strains identified as morphologically intermediate were genotyped as $L$. longiclavatum (90.0\%).

From a morphotyping perspective, $27.3 \%$ of strains initially identified as $G$. clavigera were misdiagnosed (giving a $72.7 \%$ morphotyping success rate), while from a genotyping perspective, $14.2 \%$ of the genotyped $G$. clavigera strains were mismorphotyped (genotyping success rate of $85.8 \%$ ). In contrast, $L$. longiclavatum morphotypes were rarely misidentified $(98.0 \%$ morphotyping success rate) but had the lowest genotype success rate $(58.0 \%)$ due primarily to the large number of intermediate strains. O. montium morphotypes were occasionally misidentified $(85.1 \%$ morphotyping success rate) but had the highest genotyping success rate $(95.8 \%)$.

\section{Relative Abundance}

The relative abundance of fungal species for each landscape and sampling period are summarized in Table 2. In total, 5,717 isolates were identified. Of these isolates, 2,270 (39.7\%) were G. clavigera, $2,308(40.4 \%)$ were $O$. montium, $838(14.7 \%)$ were L. longiclavatum, and 379 isolates $(6.6 \%)$ were morphologically intermediate between G. clavigera and L. longiclavatum. As described previously, MLST genotyping determined that the majority $(90 \%)$ of the strains classified as "intermediate" were identified as $L$. longiclavatum [25, Electronic Supplementary Material 3] and had similar spatial and temporal distribution as $L$. longiclavatum. Based on this evidence, we grouped all intermediate strains with $L$. longiclavatum $(n=1,139$, $19.9 \%$ ) for subsequent analyses. Using these data, we identified significant landscape-level variation in relative abundance of fungal species (Table 2, Fig. 1). In particular, the abundance of both $O$. montium and $G$. clavigera decreased in northern landscapes, although the change in $G$. clavigera abundance was greater than $O$. montium. The decrease in G. clavigera abundance in the north was accompanied by an increase in L. longiclavatum.

Using PCA, we further explored this latitudinal trend (Fig. 2). The first PCA axis (PC1) was statistically significant and represented $92.15 \%$ of the variation in relative abundance of fungal species (Fig. 2). The small amount of variance captured by the second axis (PC2$7.41 \%$ ) was not statistically significant and indicated that the ordination biplot should only be interpreted with regard to $\mathrm{PC} 1$. Fungal community structure was primarily driven by the relative abundance of $L$. longiclavatum, and this 
Table 2 Total number of galleries and species scored in each landscape and the relative abundance of each fungal species
Location and sampling period abbreviations as in Table 1

Gc, G. clavigera; Om, O. montium; $\mathrm{Ll}$, L. longiclavatum; $R A$, relative abundance

\begin{tabular}{|c|c|c|c|c|c|c|c|c|c|}
\hline & \multirow[t]{2}{*}{ Period } & \multirow[t]{2}{*}{ Total Gall } & \multirow[t]{2}{*}{ Total Morph } & \multicolumn{2}{|l|}{$G c$} & \multicolumn{2}{|l|}{$\mathrm{Om}$} & \multicolumn{2}{|l|}{$L I$} \\
\hline & & & & $n$ & RA & $n$ & RA & $n$ & RA \\
\hline \multicolumn{10}{|c|}{ North } \\
\hline FV & 1 & 86 & 180 & 54 & 0.30 & 71 & 0.39 & 55 & 0.31 \\
\hline $\mathrm{FV}$ & 2 & 306 & 654 & 201 & 0.31 & 237 & 0.36 & 216 & 0.33 \\
\hline $\mathrm{TR}$ & 2 & 272 & 569 & 211 & 0.37 & 221 & 0.39 & 137 & 0.24 \\
\hline GP & 1 & 175 & 341 & 110 & 0.32 & 124 & 0.36 & 107 & 0.31 \\
\hline GP & 2 & 274 & 567 & 179 & 0.32 & 217 & 0.38 & 171 & 0.30 \\
\hline $\mathrm{FC}$ & 1 & 60 & 129 & 41 & 0.32 & 49 & 0.38 & 39 & 0.30 \\
\hline $\mathrm{FC}$ & 2 & 88 & 148 & 69 & 0.47 & 40 & 0.27 & 39 & 0.26 \\
\hline WK & 1 & 148 & 329 & 116 & 0.35 & 132 & 0.40 & 81 & 0.25 \\
\hline WK & 2 & 184 & 397 & 115 & 0.29 & 162 & 0.41 & 120 & 0.30 \\
\hline $\mathrm{V}$ & 2 & 189 & 403 & 171 & 0.42 & 175 & 0.43 & 57 & 0.14 \\
\hline \multicolumn{2}{|c|}{ North totals } & 1,782 & 3,717 & 1,267 & & 1,428 & & 1,022 & \\
\hline \multicolumn{3}{|c|}{ North average RA } & & & 0.35 & & 0.38 & & 0.27 \\
\hline \multicolumn{10}{|c|}{ South } \\
\hline G & 2 & 176 & 356 & 165 & 0.46 & 161 & 0.45 & 30 & 0.08 \\
\hline KY & 2 & 152 & 298 & 143 & 0.48 & 131 & 0.44 & 24 & 0.08 \\
\hline $\mathrm{CA}$ & 1 & 102 & 188 & 98 & 0.52 & 82 & 0.44 & 8 & 0.04 \\
\hline $\mathrm{CA}$ & 2 & 318 & 580 & 305 & 0.53 & 248 & 0.43 & 27 & 0.04 \\
\hline SW & 2 & 203 & 392 & 190 & 0.48 & 185 & 0.47 & 17 & 0.04 \\
\hline $\mathrm{CN}$ & 1 & 28 & 49 & 24 & 0.49 & 23 & 0.47 & 2 & 0.04 \\
\hline $\mathrm{CN}$ & 2 & 70 & 113 & 67 & 0.59 & 39 & 0.35 & 7 & 0.06 \\
\hline $\mathrm{CH}$ & 2 & 21 & 24 & 11 & 0.46 & 11 & 0.46 & 2 & 0.08 \\
\hline \multicolumn{2}{|c|}{ South totals } & 1,070 & 2,000 & 1,003 & & 880 & & 117 & \\
\hline \multicolumn{2}{|c|}{ South average RA } & & & & 0.50 & & 0.44 & & 0.06 \\
\hline Totals & & 2,852 & 5,717 & 2,270 & & 2,308 & & 1,139 & \\
\hline
\end{tabular}

trend was strongly and significantly associated with increasing latitude, based on our post-hoc fitted vector of latitude values $\left(R^{2}=0.8159, p=0.001\right.$; Fig. 2$)$. In contrast, the relative abundance of $G$. clavigera, and to a lesser degree $O$. montium, was associated with elevation $\left(R^{2}=\right.$ 0.452, $p=0.001$; Fig. 2). The northern and southern landscape groups were resolved by PC1 (Fig. 2), with the exception of V.bb and V.cc, two northern sites that cluster more closely with southern sites, rather than other northern sites. As hypothesized, L. longiclavatum became more abundant as latitude increased and $G$. clavigera decreased in abundance as latitude increased, although this latter trend appeared less pronounced and also appeared related to increased elevation in the southern region of the study area. $O$. montium did not correlate as strongly with latitude as $L$. longiclavatum and $G$. clavigera but was nonetheless associated with southern sample locations (right quadrants of ordination; Fig. 2. PCA also identified several unique sample sites. A single site in Crowsnest Pass in 2008 (CN. bb) showed unique composition relative to the rest of the sites (Fig. 3). This site had greater than expected relative dominance by $G$. clavigera and had a similar reduction in O. montium.

\section{Rates of Change}

Three mixed-effects regression models were built to describe relative abundance as a function of latitude and elevation for each species (Fig. 3; Table 3). Both G. clavigera and $L$. longiclavatum were strongly affected by changes in latitude; G. clavigera abundance decreased with latitude and, conversely, L. longiclavatum abundance increased with latitude. $O$. montium was also affected by changes in latitude, although this relationship was not as strong as was observed in the other species (Table 3). Relative abundance of $G$. clavigera was influenced by the interaction between latitude and elevation, although this effect was weak relative to that of latitude (Table 3). Neither elevation alone nor in combination with latitude helped explain variation in the relative abundance of $O$. montium or L. longiclavatum.

Three mixed-effect ANCOVA tests were used to assess the differences between the slopes of the modeled relation- 
Figure 2 Principle component biplot of proportional fungal composition across 50 sample sites in Alberta and British Columbia. Black text represents sample sites. Sample region (landscape-as in Fig. 1) is indicated by the capital letters to the left of the period in the text labels, while characters to the right of the period represent site IDs (collections of trees) within those regions. Double characters (e.g., " $a$ a") were sites collected in period 2, while single characters (e.g., " $a$ ") were sites collected in period 1 . Species proportions are represented by black vectors. Gray vectors represent fitted vectors of latitude $\left(R^{2}=0.816 ; p=0.001\right)$ and elevation $\left(R^{2}=0.452 ; p=\right.$ $0.001)$ that indicate the strength of correlation of these factors with the ordination. Italicized labels in the lower left and right quadrants of the ordination indicate directional association of sites with latitude. Gc, G. clavigera; Ll, L. longiclavatum; Om, O. montium

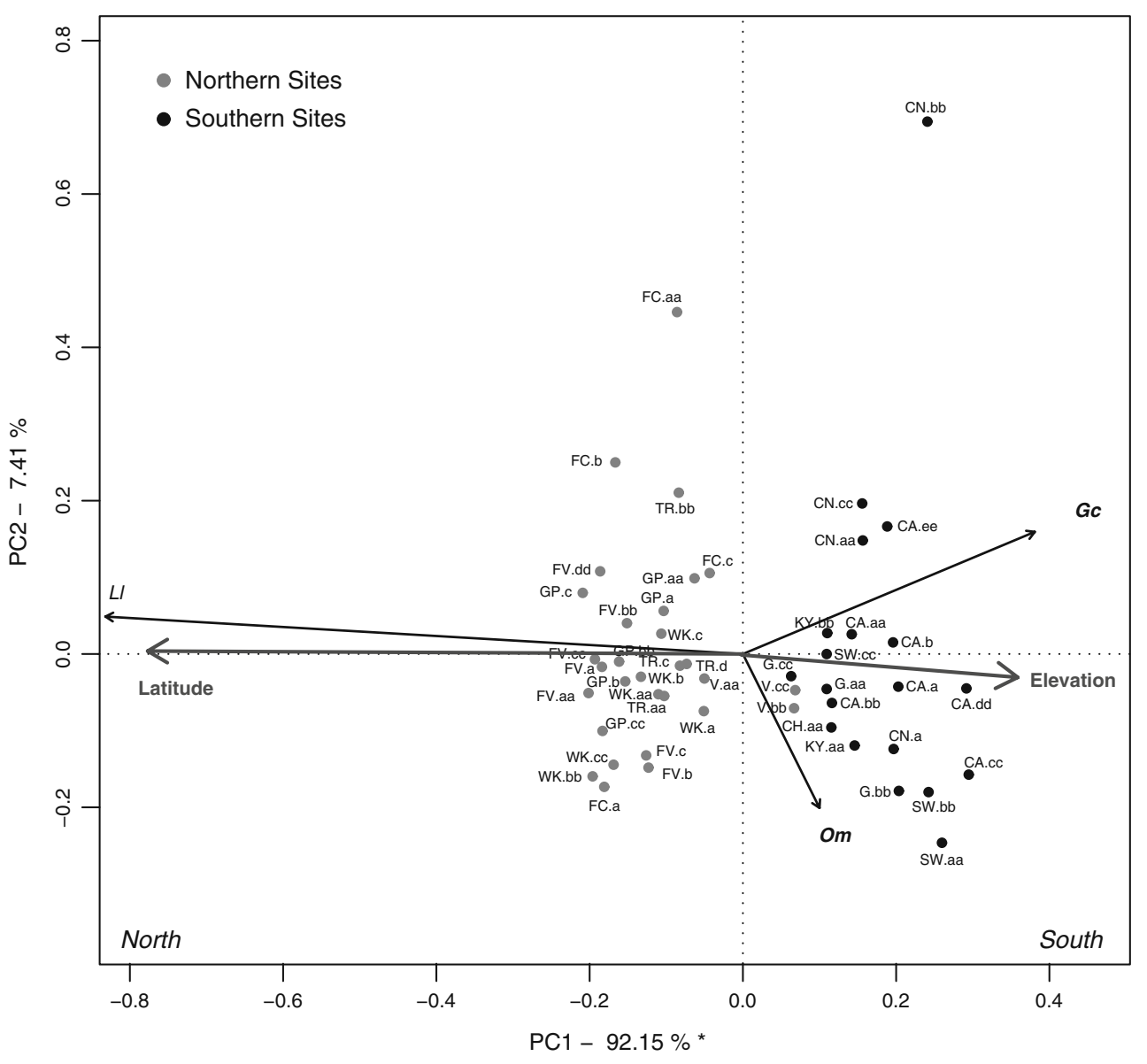

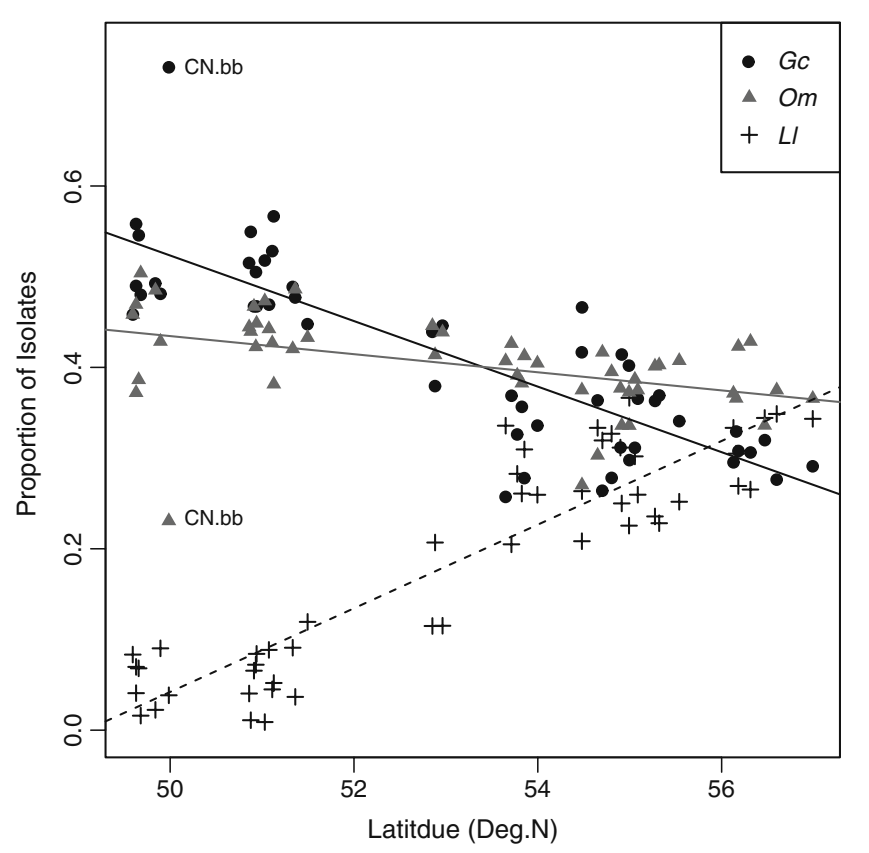

Figure 3 Relative abundance of each species as a function of latitude. Each point represents the percentage of each species at a particular site calculated as a proportion of total number of isolates for each location. Straight lines are linear regression lines fit by least squares. Sample site labels as in Fig. 2. Gc, G. clavigera; Om, O. montium; Ll, L. longiclavatum ships between species abundance and latitude. The absolute rates of change in G. clavigera and L. longiclavatum in response to latitude were not significantly different based on examination of the interaction term between species and latitude (ANCOVA; $F=3.48, p=0.065$ ) and suggested symmetrical replacement along the sampled latitudinal gradient. The slopes of the models for $G$. clavigera and $O$. montium were different (ANCOVA; $F=34.04 ; p<0.001$ ), as were those for L. longiclavatum and O. montium (ANCOVA; $F=46.18, p<0.001)$. We did not examine differences in each species' response to elevation because of the lack of a strong and consistent response to elevation for all species, and the strong negative correlation between latitude and elevation $(r=-0.74)$ which indicated that the two covariates represent a very similar gradient.

\section{Temporal Variation}

Some differences in relative abundance were observed between years (Fig. 4). In general, more isolates were identified as $G$. clavigera in period 2 than in period 1 , and similarly fewer isolates were identified as L. longiclavatum. No change was observed in $O$. montium between years. Despite these trends, the effect of sampling period on abundance was not significant for either $G$. clavigera 
Table 3 Summary of mixedeffect regression models describing relative abundance of each morphotype as a function of latitude

\begin{tabular}{|c|c|c|c|c|c|c|c|}
\hline Model & Species & LogLik & Parameter & Value & Std. error & $F$ & $p$ \\
\hline \multirow[t]{4}{*}{1} & \multirow[t]{4}{*}{$G c$} & \multirow[t]{4}{*}{63.208} & Intercept & 0.389 & 0.015 & 697.875 & 0.000 \\
\hline & & & Latitude & -0.079 & 0.014 & 30.753 & 0.000 \\
\hline & & & Elevation & 0.002 & 0.014 & 0.022 & 0.884 \\
\hline & & & Lat. $\times$ Elev. & -0.030 & 0.015 & 4.156 & 0.049 \\
\hline \multirow[t]{4}{*}{2} & \multirow[t]{4}{*}{$\mathrm{Om}$} & \multirow[t]{4}{*}{69.500} & Intercept & 0.411 & 0.014 & 865.466 & 0.000 \\
\hline & & & Latitude & 0.028 & 0.013 & 4.229 & 0.047 \\
\hline & & & Elevation & 0.003 & 0.013 & 0.046 & 0.832 \\
\hline & & & Lat. $\times$ Elev. & 0.011 & 0.014 & 0.666 & 0.420 \\
\hline \multirow[t]{4}{*}{3} & \multirow[t]{4}{*}{$L I$} & \multirow[t]{4}{*}{71.595} & Intercept & 0.198 & 0.015 & 183.331 & 0.000 \\
\hline & & & Latitude & 0.104 & 0.014 & 55.168 & 0.000 \\
\hline & & & Elevation & -0.001 & 0.013 & 0.003 & 0.956 \\
\hline & & & Lat. $\times$ Elev. & 0.017 & 0.014 & 1.509 & 0.227 \\
\hline
\end{tabular}

(ANCOVA; $F=0.699, p=0.407$ ) or $L$. longiclavatum (ANCOVA; $F=0.47, p=0.4933$ ).

\section{Species Coexistence}

The relative proportion of species associations within MPB galleries varied spatially (Table 4; Fig. 5). Of the galleries surveyed, nearly all contained at least one ophiostomatoid fungal species (99.97\%), with the majority containing two species (Table 4, Fig. 5). The most common association occurred between $G$. clavigera and $O$. montium ( $G$. clavigera-O. montium, 46\%; Table 4, Fig. 5). Cypress Hills was the exception where the majority of galleries contained only a single species (G. clavigera; Fig. 5).

Within the entire study area, the frequencies of the three possible pair-wise combinations were different from each other $\left(X^{2}=610.69, d f=2, p<0.001\right)$ given expected frequencies based on species abundances. Similar results were found for both northern $\left(X^{2}=220.13, d f=3, p<0.001\right)$ and southern regions $\left(X^{2}=224.26, d f=2, p<0.001\right)$. Almost all paired comparisons were also significant. The one exception to this was in the southern region where the frequency of the G. clavigera-L. longiclavatum combination was not different than that of $O$. montium-L. longiclavatum $(2.3 \%$ vs. $2 \% ; X^{2}=0.03, d f=1, p=0.86$ ). However, both combinations were different from expected given individual abundances. In fact, we found that the frequency of each individual combination was significantly different than expected (all $X^{2}>30, p<0.001$ ) given the relative abundances of each species, with the exception of the O. montium-L. longiclavatum pairing in the northern region $\left(X^{2}=1.96\right.$, $d f=2, p=0.16$ ).

Similar to the results described above, we also found that the proportions of each pair-wise combination were different between northern and southern regions (all $X^{2}>$ $100, p<0.001)$.

In sites belonging to the southern group $(\mathrm{CA}, \mathrm{CH}, \mathrm{CN}$, $\mathrm{G}, \mathrm{KY}, \mathrm{SW}$ ) where L. longiclavatum was relatively rare (abundance $<20 \%$ ), G. clavigera-O. montium is by far the most common species combination in galleries (Fig. 5). In the northern group of sites (FC, FV, GP, TR, V, WK) where L. longiclavatum was more common (abundance $>20 \%$ ), a different species coexistence pattern was observed. The association between $G$. clavigera-O. montium was less common $\left(X^{2}=195, d f=2, p<0.001\right)$, while the associations that included $L$. longiclavatum significantly increased in
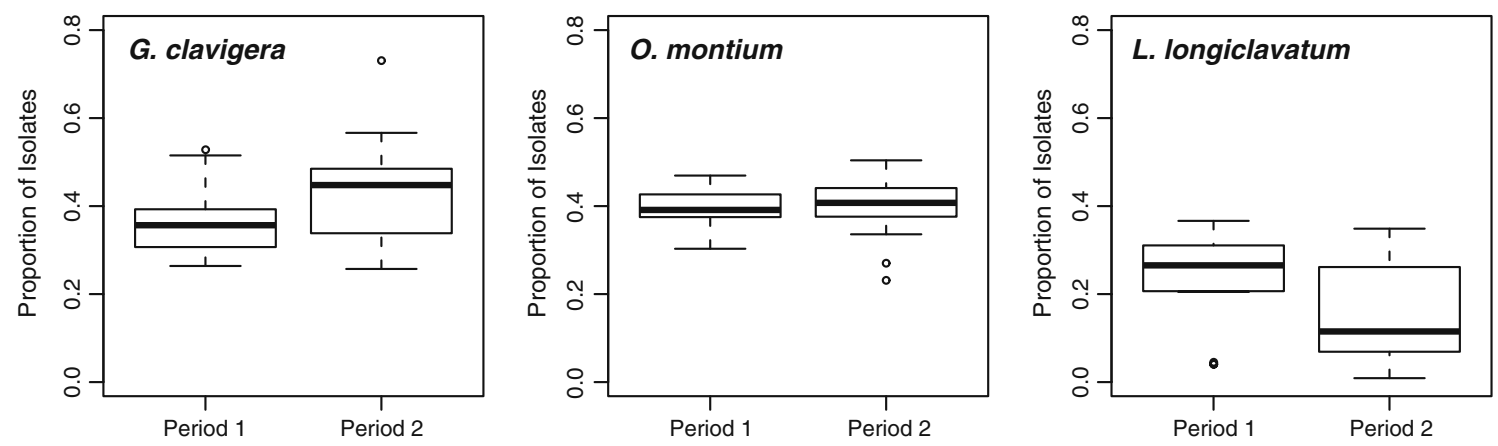

Figure 4 Boxplots illustrating differences in relative abundance for each species between sampling periods 

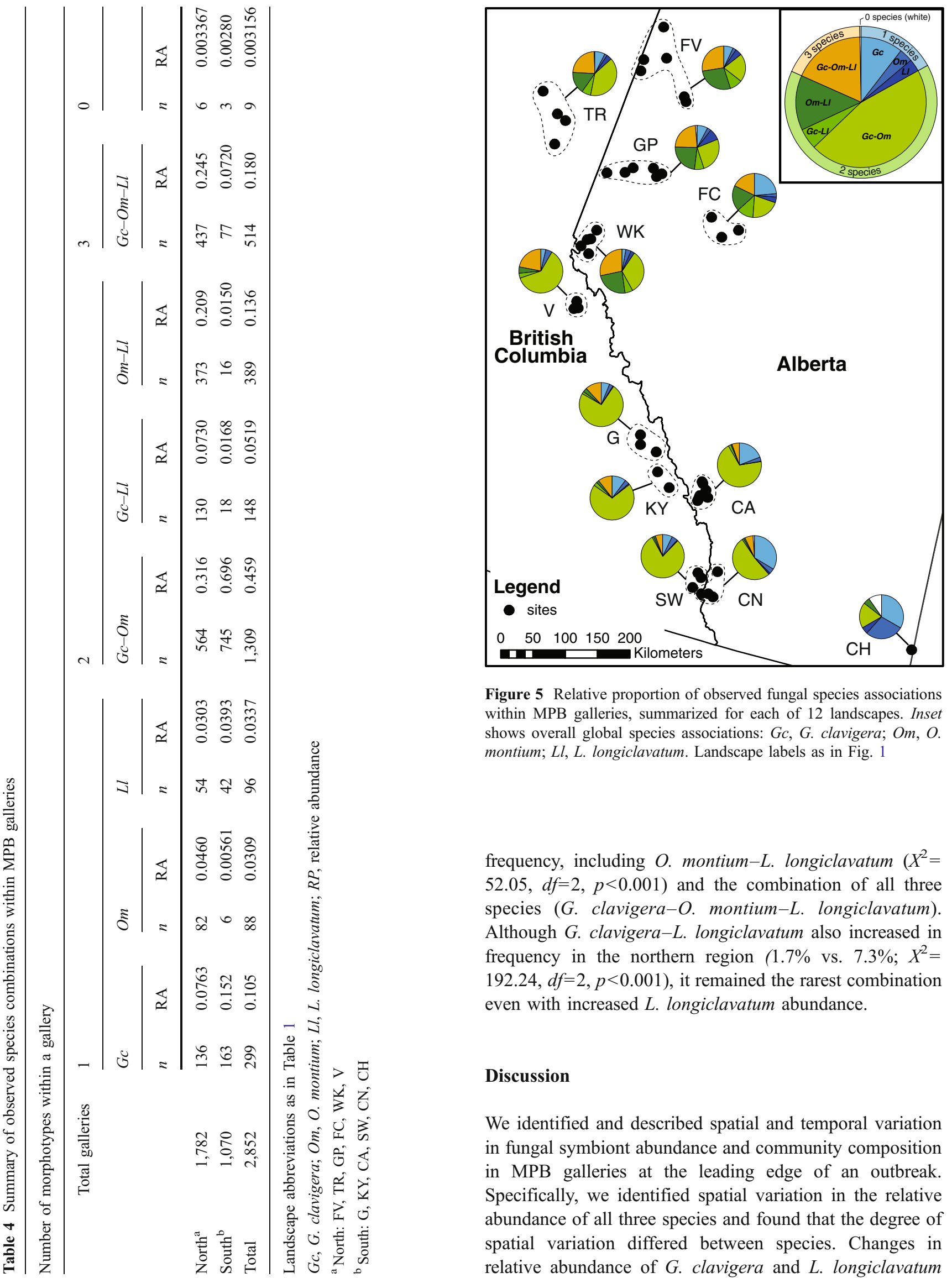

Figure 5 Relative proportion of observed fungal species associations within MPB galleries, summarized for each of 12 landscapes. Inset shows overall global species associations: Gc, G. clavigera; Om, O. montium; $\mathrm{Ll}$, L. longiclavatum. Landscape labels as in Fig. 1

frequency, including $O$. montium-L. longiclavatum $\left(X^{2}=\right.$ $52.05, d f=2, p<0.001)$ and the combination of all three species ( $G$. clavigera-O. montium-L. longiclavatum). Although G. clavigera-L. longiclavatum also increased in frequency in the northern region $\left(1.7 \%\right.$ vs. $7.3 \% ; X^{2}=$ 192.24, $d f=2, p<0.001$ ), it remained the rarest combination even with increased $L$. longiclavatum abundance.

\section{Discussion}

We identified and described spatial and temporal variation in fungal symbiont abundance and community composition in MPB galleries at the leading edge of an outbreak. Specifically, we identified spatial variation in the relative abundance of all three species and found that the degree of spatial variation differed between species. Changes in relative abundance of $G$. clavigera and $L$. longiclavatum 
were strongly determined by latitude, while changes in $O$. montium were less apparent. Elevation did no account for additional variation in fungal abundance in $O$. montium and L. longiclavatum but did weakly influence the abundance of G. clavigera in combination with latitude. We used latitude and elevation as simple proxies for climatic conditions, similar to what has been done previously in other studies of fungal diversity [e.g., 35-37]. Although high elevation and low latitude have potentially different temperature-related consequences, we were unable to fully examine these factors independently due to their correlated nature, as well as the nature of our sampling scheme that fell along this gradient.

\section{Fungal Abundances}

When we compared the observed variation in fungal abundance with previously published accounts, a landscapescale pattern emerged. In earlier studies, the majority of work occurred in southern MPB populations where L. longiclavatum was rarely encountered [12, 18]. Our results, in congruence with Rice and Langor [18], indicate that $L$. longiclavatum becomes more abundant in northern sites (Figs. 2 and 3). By comparing the slopes of the relative abundance of the three species, we observed a gradual change in abundance with increasing latitude in this species. Furthermore, a symmetrical and opposite rate of change was also observed in $G$. clavigera. The symmetry of these two slopes (Fig. 3) suggests that G. clavigera is being replaced by $L$. longiclavatum as latitude increases, in turn suggesting competitive exclusion of one species by the other. While both $G$. clavigera and $O$. montium decrease in abundance with increasing latitude, the rate of change in $O$. montium was less pronounced. This finding was contrary to our initial expectations. Given that previous work has shown $O$. montium to be temperature sensitive in Montana and Idaho [7] and with a higher optimal growth temperature and slower growth at cool temperatures than $G$. clavigera $[19,20]$, we expected that $O$. montium would show a similar or greater rate of decreasing abundance with increasing latitude than $G$. clavigera. However, our findings do support other observations that in northern regions $O$. montium demonstrates variability in cold tolerance and may be more cold tolerant than initially thought [18].

\section{Fungal Community Composition}

The symmetrical rates of change and apparent replacement of $G$. clavigera by $L$. longiclavatum at northern latitudes suggest that processes of competitive exclusion are influencing patterns of fungal community composition. This is further supported by the observation that $G$. clavigera and L. longiclavatum are rarely paired together within a gallery
(Table 4), while the combination of $G$. clavigera and $O$. montium, and $O$. montium and L. longiclavatum are more common (Table 4). Furthermore, in almost all cases, the relative frequencies of the possible pair-wise combinations are different from what is expected given the raw abundances of the species. This indicates non-random associations between fungal symbionts that vary spatially. Interestingly, the one exception to this pattern is the $O$. montium-L. longiclavatum combination in the north, the frequency of which was proportional to the frequency of each individual species.

Phylogenetically, L. longiclavatum and G. clavigera are closely related while $O$. montium is more distantly related [38, 39]. G. clavigera and L. longiclavatum are also morphologically similar and have similar physiological requirements, such as similar growth temperature optima $[12,13,19,40]$, thereby increasing the potential competitive interactions between these two species. Conversely, fine-scale differences in resource use may reduce competition between O. montium and G. clavigera [21]. Given these traits, G. clavigera and $L$. longiclavatum may be unable to coexist due to significant niche overlap, while $O$. montium is dissimilar enough to allow coexistence with either species, although in vitro competition experiments to examine all of these fungal combinations have not yet been conducted. Interestingly, while G. clavigera and L. longiclavatum rarely occur together as a pair, all three species together are able to coexist (Table 4). We hypothesize that the addition of the third species, $O$. montium, may create a niche space that allows all three species to coexist, a natural example of a "rock-paper-scissors" means of coexistence [41]. In a "rock-paper-scissors" scenario, competition between pairs of species results in one species outcompeting the other. However, in this three-species system, it is possible that local competition creates additional niche space that, in combination with dispersal and repeated invasions, facilitates coexistence and the maintenance of fungal diversity [41]. Functionally, the maintenance of this fungal diversity could be essential to persistence and stability of mountain pine beetle populations across a broad range of climatic conditions. In vitro growth experiments that examine the interactions between different fungal symbionts, similar to work previously conducted on $G$. clavigera and O. montium [21], would be an ideal approach to test the hypothesis of a "rock-paper-scissors" means of coexistence and to further explore patterns of competition and coexistence among these fungi.

Temporal Variation

Although we did not find significant differences in species abundances between years, we did observe some interesting temporal trends in $G$. clavigera and $L$. longiclavatum 
(Fig. 4). We also identified unexplained variation in fungal abundance at some individual sites (i.e., CN.bb; Fig. 3). Variation between sample periods and among sites in our data may also be a product of weather, dispersal patterns, or local environmental factors (e.g., temperature or elevation). Based on survey data, we know that some populations, particularly those in northeastern $\mathrm{BC}$ and northwestern $\mathrm{AB}$ may have originated from long-distance dispersal events [6]. These migrant populations may be associated with different fungal communities, impacting year-to-year differences in fungal composition at sites experiencing repeated long-distance colonization. Uncertainty in the relative roles of these different factors highlights the need for further research into beetle movement and patterns of gene flow in both the beetle and its fungal associates as a function of spatial and temporal variation. Furthermore, although previous work observed changes in fungal abundance over time in a single season $[17,40]$, our results indicate that it may be useful to track these changes over longer time periods. This will be particularly important as MPB populations expand into novel environments such as the jack pine forests in northern Alberta, or as they shift from epidemic to endemic levels.

\section{Potential Sources of Bias}

By standardizing our sampling methodologies (e.g., cool period sampling, room temperature incubation), we may have introduced systematic bias into our assessment of fungal community structure, for example, favoring fastergrowing and prolifically sporulating fungal species over slower-growing species, or characterizing the fungal community composition associated with cool weather as opposed to warm weather conditions. With this potential bias in mind, it is interesting to note that the less-prolific sporulating species (O. montium) is quite abundant and shows less response to latitude than either of the two rapidly sporulating fungal species ( $G$. clavigera and $L$. longiclavatum). Given this pattern, we are confident that the patterns observed reflect actual trends in community structure rather than an artifact of our collection and isolation practices.

By contrasting the morphotyping results with the MLST genotypes of a subset of isolates, we identified additional potential biases in our surveys that could also affect other ecologically based studies of these fungi. First, the vast majority of strains identified by morphological characteristics as "intermediate" between $L$. longiclavatum and $G$. clavigera were actually L. longiclavatum. Here, the availability of molecular data provided objective justification for their inclusion with $L$. longiclavatum count data in subsequent analyses. Second, MLST data suggest that the actual fungal proportions associated with MPB are likely overestimated for $G$. clavigera and $O$. montium and underestimated for $L$. longiclavatum proportions due to morphological misdiagnoses. However, it is likely that these biases would augment the patterns observed in the morphologically diagnosed data rather than diminish them. Third, the two additional fungal associates, $G$. aurea and $L$. terebrantis, identified using MLST data were not identified morphologically but appear to form a very small proportion of the overall fungal symbiont fauna associated with MPB. Their inclusion, had they been reliably distinguishable by morphological characteristics likely would not affect our inferences regarding the relationships between geographical location and fungal community composition. Together, these observations point to the utility of applying DNAbased identification techniques [25, 42-44] to the mixedspecies environmental cultures that were obtained to confirm species identifications and to test for cryptic species assemblages.

\section{Conclusions}

We identified significant biogeographic patterns of community composition in the major mountain pine beetle associated fungal symbionts. The observed patterns of species coexistence and competitive exclusion among fungi suggest that complex ecological processes are controlling community structure [45]. As in other bark beetle systems, variation in fungal symbiont associations [9, references therein] has been linked to environmental factors and substrate variability [7, 46-49]. Dynamics and interactions between co-occurring bark beetle symbionts have also been explored [46, 50-52]. This study provides one of the first large-scale surveys of symbiont composition patterns in natural MPB populations, giving insight to landscapelevel processes impacting symbiont community dynamics. Understanding the biotic and abiotic factors that lead to symbiont community variability over time and space is imperative as the symbiont community can impact beetle colonization success and population dynamics [9, 48, 53].

To further understand the mechanisms behind the patterns of variation in fungal symbionts, it will be important to link fungal abundance and community composition to additional environmental features (e.g., fungal "habitat") and to examine the persistence of these trends throughout the life cycle of the beetle. Furthermore, explicit quantification of the interactions between the three primary fungal symbionts through growth experiments on artificial media, particularly under varying temperature regimes, are required to determine the mechanisms that underpin the observed biogeographic patterns of MPB fungal community changes. Variation in fungal symbiont assemblages across a range of environmental conditions 
represents an important element of MPB functional diversity that, in combination with intraspecific variation within the symbionts and host, will be important to understand the success of MPB attacks in novel habitats and to predict future risk of outbreak.

Acknowledgments First, we wish to thank the four anonymous reviewers for their comments and suggestions that helped improve this manuscript. We wish to acknowledge all the hard work and dedication of the TRIA field and laboratory technicians who obtained and processed fungal samples. We are grateful for the time and support provided by Sepideh Massoumi Alamouti, Colette Breuil, Richard Hamelin, and Dave Langor, whose advice and insight have been invaluable. Funding for this research has been provided through grants from the Government of Alberta (AAET/AFRI-859-G07), as well as from the Government of Alberta through Genome Alberta, and from the Government of British Columbia through Genome BC (The TRIA Project, http://www. thetriaproject.ca) to JEKC, DWC, and FAHS. PMAJ was also supported by a Killam Post-Doctoral Fellowship at the University of Alberta.

Open Access This article is distributed under the terms of the Creative Commons Attribution Noncommercial License which permits any noncommercial use, distribution, and reproduction in any medium, provided the original author(s) and source are credited.

\section{References}

1. Klepzig KD, Adams AS, Handelsman J, Raffa KF (2009) Symbioses: a key driver of insect physiological processes, ecological interactions, evolutionary diversification, and impacts on humans. Environ Entomol 38:67-77

2. Relman DA (2008) 'Til death do us part': coming to terms with symbiotic relationships. Nat Rev Microbiol 6:721-724

3. Six DL, Klepzig KD (2004) Dendroctonus bark beetles as model systems for studies on symbiosis. Symbiosis 37:207-232

4. Taylor SW, Carroll AL, Alfaro RI, Safranyik L (2006) Forest, climate, andmountain pine beetle outbreak dynamics in western Canada. In: Safranyik L, Wilson WR (eds) The mountain pine: a synthesis of biology, management, and impacts on lodgepole pine. Natural Resources Canada, Canadian Forest Service, Pacific Forestry Centre, Victoria, pp 67-94

5. Raffa KF, Aukema BH, Bentz BJ, Carroll AL, Hicke JA, Turner MG, Romme WH (2008) Cross-scale drivers of natural disturbances prone to anthropogenic amplification: the dynamics of bark beetle eruptions. Bioscience 58:501-517

6. Safranyik L, Carroll AL, Régniére J, Langor DW, Riel WG, Shore TL, Peter B, Cooke BJ, Nealis VG, Taylor SW (2010) Potential for range expansion of mountain pine beetle into the boreal forest of North America. Can Entomol 142:415-441

7. Six DL, Bentz BJ (2007) Temperature determines symbiont abundance in a multipartite bark beetle-fungus ectosymbiosis. Microb Ecol 54:112-118

8. Bleiker KP, Six DL (2007) Dietary benefits of fungal associates to an eruptive herbivore: potential implications of multiple associates on host population dynamics. Environ Entomol 36:1384-1396

9. Lieutier F, Yart A, Salle A (2009) Stimulation of tree defenses by ophiostomatoid fungi can explain attack success of bark beetles on conifers. Ann For Sci 66:22

10. Six DL (2003) A comparison of mycangial and phoretic fungi of individual mountain pine beetles. Can J For Res 33:1331-1334

11. Six DL, Paine TD (1998) Effects of mycangial fungi and host tree species on progeny survival and emergence of Dendroctonus ponderosae (Coleoptera: Scolytidae). Environ Entomol 27:1393-1401
12. Lee S, Kim JJ, Breuil C (2006) Diversity of fungi associated with the mountain pine beetle, Dendroctonus ponderosae and infested lodgepole pines in British Columbia. Fungal Divers 22:91-105

13. Lee S, Kim JJ, Breuil C (2005) Leptographium longiclavatum sp nov., a new species associated with the mountain pine beetle, Dendroctonus ponderosae. Mycol Res 109:1162-1170

14. Berryman A (1972) Resistance of conifers to invasion by bark beetle-fungus associations. Bioscience 22:598-602

15. Bentz BJ, Six DL (2006) Ergosterol content of fungi associated with Dendroctonus ponderosae and Dendroctonus rufipennis (Coleoptera: Curculionidae, Scolytinae). Ann Entomol Soc Am 99:189-194

16. Six DL (2005) Population genetics of bark beetles and their associated blue-stain fungi with the use of molecular markers. In: Lundquist J, Hamelin RC (eds) Forest pathology: from genes to landscapes. APS, St. Paul, pp 31-39

17. Adams AS, Six DL (2007) Temporal variation in mycophagy and prevalence of fungi associated with developmental stages of Dendroctonus ponderosae (Coleoptera: Curculionidae). Environ Entomol 36:64-72

18. Rice A, Langor D (2009) Mountain pine beetle-associated bluestain fungi in lodgepole $\mathrm{x}$ jack pine hybrids near Grande Prairie, Alberta (Canada). For Pathol 39:323-334

19. Rice AV, Thormann MN, Langor DW (2008) Mountain pine beetle-associated blue-stain fungi are differentially adapted to boreal temperatures. For Pathol 38:113-123

20. Solheim H, Krokene P (1998) Growth and virulence of mountain pine beetle associated blue-stain fungi, Ophiostoma clavigerum and Ophiostoma montium. Can J Bot 76:561-566

21. Bleiker KP, Six DL (2009) Competition and coexistence in a multi-partner mutualism: interactions between two fungal symbionts of the mountain pine beetle in beetle-attacked trees. Microb Ecol 57:191-202

22. Peay K, Kennedy P, Bruns T (2008) Fungal community ecology: a hybrid beast with a molecular master. Bioscience 58:799-801

23. Hopkins AD (1920) The bioclimatic law. Mon Weather Rev 48:355-355

24. Roe AD, Rice AV, Coltman D, Cooke JEK, Sperling FAH (2011) Comparative phylogeography, genetic differentiation, and contrasting reproductive modes in three fungal symbionts of a multipartite bark beetle symbiosis. Mol Ecol 20:584-600

25. Roe AD, Rice AV, Bromilow SE, Cooke JEK, Sperling FAH (2010) Multilocus species identification and fungal DNA barcoding: insights from blue stain fungal symbionts of the mountain pine beetle. Mol Ecol Res 10:946-959

26. Ecological Stratification Working Group (1996) A national ecological framework for Canada. Agri-Food Canada, Research Branch, Centre for Land and Biological Resources Research and Environment Canada, State of the Environment Directorate, Ecozone Analysis Branch, Ottawa

27. Pielou E (1984) The interpretation of ecological data. Wiley, New York

28. Legendre P, Gallagher ED (2001) Ecologically meaningful transformations for ordination of species data. Oecologia 129:271-280

29. Oksanen J, Blanchet G, Kindt R, Legendre P, O'Hara R, Simpson G, Solymos P, Stevens M, Wagner H (2010) vegan: Community Ecology Package. R package vers. 1.17-2

30. Jackson DA (1993) Stopping rules in principal components analysis: a comparison of heuristical and statistical approaches. Ecology 74:2204-2214

31. Pinheiro J, Bates D, DebRoy S, Sarkar D, and the R Development Core Team (2011) nlme: Linear and nonlinear mixed effects models. R package version 3.1-97

32. Pinheiro JC, Bates DM (2000) Mixed-effects models in S and SPLUS. Springer-Verlag, New York 
33. R Development Core Team (2011) R: A language and environment for statistical computing. R Foundation for Statistical Computing, Vienna, Austria

34. Ye TZ, Yang R-C, Yeh FC (2002) Population structure of a lodgepole pine (Pinus contorta) and jack pine (P. banksiana) complex as revealed by random amplified polymorphic DNA. Genome 45:530-540

35. Muths E, Pilliod DS, Livo LJ (2008) Distribution and environmental limitations of an amphibian pathogen in the Rocky Mountains, USA. Biol Conserv 141:1484-1492

36. Newsham KK, Upson R, Read DJ (2009) Mycorrhizas and dark septate root endophytes in polar regions. Fungal Ecol 2:10-20

37. Yergeau E, Bokhorst S, Huiskes AHL, Boschker HTS, Aerts R, Kowalchuk GA (2007) Size and structure of bacterial, fungal and nematode communities along an Antarctic environmental gradient. FEMS Microbiol Ecol 59:436-451

38. Six DL, Paine TD (1999) Phylogenetic comparison of ascomycete mycangial fungi and Dendroctonus bark beetles (Coleoptera: Scolytidae). Ann Entomol Soc Am 92:159-166

39. Massoumi Alamouti S, Tsui CKM, Breuil C (2009) Multigene phylogeny of filamentous ambrosia fungi associated with ambrosia and bark beetles. Mycol Res 113:822-835

40. Rice A, Thormann M, Langor D (2007) Virulence of, and interactions among, mountain pine beetle associated blue-stain fungi on two pine species and their hybrids in Alberta. Can J Bot 85:316-323

41. Kerr B, Riley MA, Feldman MW, Bohannan BJ (2002) Local dispersal promotes biodiversity in a real-life game of rock-paperscissors. Nature 418:171-174

42. Khadempour L, Massoumi Alamouti S, Hamelin RC, Bohlmann J, Breuil C (2010) Target-specific PCR primers can detect and differentiate ophiostomatoid fungi from microbial communities associated with the mountain pine beetle Dendroctonus ponderosae. Fungal Biol 114:825-833

43. Lee S, Kim JJ, Fung S, Breuil C (2003) A PCR-RFLP marker distinguishing Ophiostoma clavigerum from morphologically similar Leptographium species associated with bark beetles. Can J Bot 81:1104-1112
44. Tsui CKM, Wang B, Khadempour L, Alamouti SM, Bohlmann J, Murray B, Hamelin RC (2010) Rapid identification and detection of pine pathogenic fungi associated with mountain pine beetles by padlock probes. J Microbiol Meth 83:26-33

45. Gourbiere S, Gourbiere F (2002) Competition between unit-restricted fungi: a metapopulation model. J Theor Biol 217:351-368

46. Hofstetter RW, Dempsey TD, Klepzig KD, Ayres MP (2007) Temperature-dependent effects on mutualistic, antagonistic, and commensalistic interaction among insects, fungi, and mites. Community Ecol 8:47-56

47. Jankowiak R (2005) Fungi associated with Ips typographus on Picea abies in southern Poland and their succession into the phloem and sapwood of beetle-infested trees and logs. For Pathol $35: 37-55$

48. Klepzig KD, Six DL (2004) Bark beetle-fungal symbiosis: context dependency in complex associations. Symbiosis 37:189-205

49. Sallé A, Monclus R, Yart A, Garcia J, Romary P, Lieutier F (2005) Fungal flora associated with Ips typographus: frequency, virulence, and ability to stimulate host defense reaction in relation to insect population levels. Can J For Res 35:365-373

50. Aukema BH, Werner RA, Haberkern KE, Illman BL, Clayton MK, Raffa KF (2005) Quantifying sources of variation in the frequency of fungi associated with spruce beetles: implications for hypothesis testing and sampling methodology in bark beetlesymiont relationships. For Ecol Manage 10:187-202

51. Hofstetter RW, Cronin JT, Klepzig KD, Moser JC, Ayres MP (2006) Antagonisms, mutualisms and commensalisms affect outbreak dynamics of the southern pine beetle. Oecologia 147:679-691

52. Klepzig KD, Moser JC, Lombardero MJ, Ayres MP, Hofstetter RW, Walkinshaw CJ (2001) Mutualism and antagonism: ecological interactions among bark beetles, mites and fungi. In: Jeger MJ, Spence NJ (eds) Biotic interactions in plant-pathogen associations. CAB International, Wallingford, pp 237-267

53. Raffa KF, Smalley EB (1988) Response of red and Jack pines to inoculation with microbial associates of the pine engraver, Ips pini (Coleoptera: Scolytidae). Can J For Res 18:581-586 